\title{
Immunosuppressive medication is not associated with surgical site infection after surgery for intractable ulcerative colitis in children
}

\author{
Keiichi Uchida $^{1,2, *}$, Yoshikazu Ohtsuka ${ }^{2,3}$, Atsushi Yoden ${ }^{2,4}$, Hitoshi Tajiri ${ }^{2,5}$, Hideaki Kimura, \\ Takashi Isihige $^{2,7}$, Hiroyuki Yamada ${ }^{2,8}$, Katsuhiro Arai ${ }^{2,9}$, Takeshi Tomomasa ${ }^{2,10}$, Kosuke Ushijima ${ }^{2,11}$, \\ Tomoki Aomatsu ${ }^{2,4}$, Satoru Nagata ${ }^{2,12}$, Kohei Otake ${ }^{1}$, Kohei Matsushita ${ }^{1}$, Mikihiro Inoue ${ }^{1}$, \\ Takahiro Kudo $^{2,3}$, Kenji Hosoi ${ }^{2,3}$, Kazuo Takeuchi ${ }^{2,13}$, Toshiaki Shimizu ${ }^{2,3}$ \\ ${ }^{1}$ Department of Gastrointestinal and Pediatric Surgery, Mie University Graduate School of Medicine, Mie, Japan; \\ ${ }^{2}$ Members of the Japanese Society for Pediatric Inflammatory Bowel Disease Working Group; \\ ${ }^{3}$ Department of Pediatrics and Adolescent Medicine, Juntendo University Graduate School of Medicine, Tokyo, Japan; \\ ${ }^{4}$ Department of Pediatrics, Osaka Medical College, Osaka, Japan; \\ ${ }^{5}$ Department of Pediatrics, Osaka General Medical Center, Osaka, Japan; \\ ${ }^{6}$ Inflammatory Bowel Disease Center, Yokohama City University Medical Center, Kanagawa, Japan; \\ ${ }^{7}$ Department of Pediatrics, Gunma University Graduate School of Medicine, Gunma, Japan; \\ ${ }^{8}$ Department of Pediatric Gastroenterology, Nutrition and Endocrinology, Osaka Medical Center and Research Institute for \\ Maternal and Child Health, Osaka, Japan; \\ ${ }^{9}$ Division of Gastroenterology, National Center for Child Health and Development, Tokyo, Japan; \\ ${ }^{10}$ PAL Children's Clinic, Gunma, Japan; \\ ${ }^{11}$ Department of Pediatrics, Kurume University Medical Center, Fukuoka, Japan; \\ ${ }^{12}$ Department of Pediatrics, Tokyo Women's Medical University Hospital, Tokyo, Japan; \\ ${ }^{13}$ General Health Support Center, Gunma University Graduate School of Medicine, Gunma, Japan.
}

Summary Pediatric ulcerative colitis (UC) sometimes progresses to an intractable condition for medical therapy. The surgical management of $\mathrm{UC}$ is challenging because of difficult procedures and frequent infectious complications. The aim of this study was to survey surgical procedures and infectious complications in pediatric patients with $\mathrm{UC}$ in Japan and to assess the relationship between preoperatively administered immunosuppressive drugs and postoperative surgical site infection (SSI). A survey of pediatric patients treated from 2000 to 2012 was sent to 683 facilities nationwide. Secondary questionnaires were sent to physicians who followed up patients with UC who had undergone surgery with the aim of assessing the relationships between postoperative SSI and selected preoperative patient characteristics, disease severity, medications, and operative procedures. Data for 136 patients (77 boys and 59 girls) were assessed. Median age at surgery was 14.1 years (range: $2.4-18.9$ years). Surgery was performed in one stage in 35 cases, two stages in 57 cases, and three stages in 44 cases. SSI occurred in $36 / 136$ patients $(26 \%)$. According to multiple logistic regression analysis, there were statistically significant associations between SSI and staged surgery (three/one, OR: 6.7, 95\% CI: 2.1-25.5, $p=0.0007$; three/two, OR: $3.4,95 \%$ CI: $1.4-8.6, p=0.0069)$ and female sex (OR: $2.3,95 \%$ CI: $1.0-5.4, p=0.0434)$. Preoperative medications and incidence of SSI were not significantly associated. Preoperative immunosuppressive medication does not affect the incidence of SSI. Three-stage surgery and female sex are independent predictors of development of postoperative SSIs in pediatric patients with $\mathrm{UC}$.

Keywords: Ulcerative colitis, children, colectomy, complication

Released online in J-STAGE as advance publication April 16, 2017.
*Address correspondence to:

Dr. Keiichi Uchida, Department of Gastrointestinal and Pediatric Surgery, Mie University Graduate School of Medicine, 2-174 Edobashi, Tsu, Mie 514-8507, Japan.

E-mail: ucchie@clin.medic.mie-u.ac.jp 


\section{Introduction}

Inflammatory bowel diseases (IBDs), a group of chronic inflammatory gastrointestinal disorders, include Crohn disease and ulcerative colitis (UC). In recent years, the incidence of IBD has increased markedly in both adults and children. IBD's onset during childhood is in $20-30 \%$ of patients and this condition is characterized by extensive intestinal involvement and rapid early progression. IBD is more severe in children than in adults (1).

Colectomy is uncommonly performed for pediatric $\mathrm{UC}$; rates have remained stable from 1983 to 2009. However, the rates of in-hospital postoperative complications have increased, particularly in patients undergoing emergency colectomy $(2,3)$. Such complications reportedly occur in $25 \%$ of cases, gastro-intestinal (13\%) and infectious (9.3\%) complications being the most common (2). Independent predictors of postoperative complications were identified in these studies and it was concluded that optimizing timing of colectomy may reduce postoperative complications in pediatric patients with UC. Knod et al. (4) have reported that colectomy with ileoanal anastomosis does not have a greater incidence of complications in young children $(\leq 11$-years-old) with UC than in older patients and achieves a good postoperative quality of life and stool patterns.

Generally, patients with UC undergoing elective colectomy have high rates of surgical site infection (SSI), specifically deep and organ/space infections (5). Uchino et al. (6) have also demonstrated that, in surgical patients with clean-contaminated wounds, IBD is an independent risk factor for incisional SSI.

Recently, immunomodulators and biologics that affect patients' immune systems have been increasingly used to treat steroid-dependent and -resistant pediatric UC. A European Crohn's and Colitis Organisation paper has stated that preoperative thiopurines do not increase the risk of postoperative complications in adult patients (7). Additionally, there does not appear to be a higher rate of postoperative complications after colectomy for UC performed immediately following or in the medium term after the use of cyclosporine. Two studies have demonstrated that previous exposure to azathioprine/6mercaptopurine does not affect the rates of short term and late postoperative complications after pouch surgery for UC $(8,9)$. However, there are few studies concerning the relationship between preoperative drugs and postcolectomy infectious complications in children.

The aim of this study was to survey surgical procedures and postoperative infectious complications in patients with pediatric UC in Japan and to assess the relationship between preoperatively administered drugs and postoperative SSI.

\section{Materials and Methods}

This study was a retrospective analysis of data from multiple institutions in Japan. A national survey of immunomodulators and biologics used in pediatric patients with IBD (aged $<17$ years at diagnosis) between 2000 and 2012 was sent to 683 facilities treating pediatric patients with IBD in Japan from December 2012 to March 2013 (10). All participating physicians followed the Japanese diagnostic criteria for Crohn disease and UC (11). These diagnostic criteria are substantially the same as the Port criteria published by the IBD working group of the European Society for Paediatric Gastroenterology, Hepatology and Nutrition (12). This primary survey assessed the total number of pediatric patients with IBD, the number treated with immunomodulators and biologics, and the number who underwent surgery.

Secondary questionnaires were sent to physicians who followed up patients with UC who had undergone surgery, the purpose being to assess the following factors: selected patient characteristics, severity of UC, preoperative medications, operative procedure, and SSI. Patient characteristics assessed included sex, age at disease onset, age at surgery, preoperative duration of disease, indications for surgery, and preoperative blood analysis data including serum albumin, hemoglobin and C-reactive protein (CRP) concentrations.

Clinical severity of disease was evaluated according to the Pediatric Ulcerative Colitis Activity Index (PUCAI) (13) and Japanese disease severity criteria as established by the Research Committee on Inflammatory Bowel Disease of the Ministry of Health and Welfare in Japan in 1994 (11). Extent of disease at surgery was evaluated according to the Montreal classification of UC as E1 (ulcerative proctitis), E2 (left-sided UC), and E3 (extensive UC) (14).

Preoperative medical therapy assessed included systemic prednisolone (PDN), 5-aminosalicylates, azathioprine (AZA), cyclosporine A (CYA), tacrolimus (TAC), infliximab (IFX), and apheresis therapy. Patients' records were reviewed if any of these therapies had been used within 90 and 30 days prior to the first-stage operation. The dosage of PDN administered during the month before surgery was also calculated. Serum trough levels of TAC, CYA, and IFX and 6-thioguanine nucleotide concentrations in patients receiving AZA were not monitored.

Generally, operative procedures for UC both adults and children were classified as one-, two-, or threestage procedures to complete total proctocolectomy with ileal J-pouch anal anastomosis (IPAA). The one-stage procedure is total proctocolectomy with IPAA without a diversion stoma. The two-stage procedure includes total proctocolectomy with IPAA and creation of diversion ileostomy in the first stage and closure of the ileostomy in the second stage. The three-stage procedure includes subtotal colectomy with creation of a diversion ileostomy and mucus fistula of the sigmoid colon or rectal stump in the first stage. In the second stage, remnant proctocolectomy and IPAA with recreation of diversion 
ileostomy are performed. Finally, the ileostomy is closed in the third stage.

SSI was defined according to the definition of the Centers for Disease Control and Prevention as infections occurring within 30 days after surgery (15). All patients were followed up for a minimum of 30 days after the final stage of their procedures. SSI, including incisional wound infection (I-SSI), and/or organ/space infection (OS-SSI), was evaluated. Anastomotic leaks and abscesses (OS-SSI) were diagnosed by contrast imaging, computed tomography, or intraoperative findings (16).

The relationships between preoperative factors, including administered drugs, and incidence of postoperative SSI occurrence after first stage operations on pediatric patients with UC was assessed in this study.

The data were analyzed by the $\chi^{2}$ and Mann-Whitney $\mathrm{U}$ tests and by multiple logistic regression analysis using the JMP version 7 software program (SAS Institute, Cary, $\mathrm{NC}, \mathrm{USA}$ ). A $p$ value of less than 0.05 was considered to be statistically significant.

This study was approved by the institutional ethical committees of Juntendo University and all participating institutions.

\section{Results}

In the primary survey, 1,617 pediatric patients with UC were enrolled from 1 January 2000 to 31 December 2012. Data for 146 patients who had undergone surgery were collected via secondary questionnaires that were sent to the physicians who were following them up. Finally, we were able to assess 136 patients ( 77 boys and 59 girls), 10 of these patients being excluded because of incomplete data concerning surgery, medications, and SSI.

Table 1 shows the study of patients' baseline characteristics. Median age at disease onset was 12.0 years (range 0.3-17.7 years), median age at surgery 14.1 years (range: $2.4-18.9$ ), and median preoperative duration of disease 679 days (range 16-3,611 days). Median preoperative serum albumin, hemoglobin, and CRP concentrations were $3.2 \mathrm{~g} / \mathrm{dL}$ (range: $1.4-4.8 \mathrm{~g} / \mathrm{dL}$ ), $10.6 \mathrm{~g} / \mathrm{dL}$ (range: $4.9-17.5 \mathrm{~g} / \mathrm{dL}$ ), and $0.33 \mathrm{~g} / \mathrm{dL}$ (range: 0.0-16.11 g/dL), respectively. As to preoperative disease activity, median PUCAI score was 45 (range: 0-85). According to Japanese criteria of disease severity, 12\% of patients had mild disease, $40 \%$ moderate, and $45 \%$ severe. According to the Montreal classification, $96 \%$ of patients had E3 disease (extensive UC).

Indications for surgery, which overlapped each other in this study, are listed in Table 2. The most frequent indications for surgery were intractable disease caused by resistance to medications, including steroid dependency and steroid resistance, in $77 \%$ of patients. The next most frequent indications were poor quality of life in $23 \%$, adverse effects of drugs in $12 \%$, massive bleeding in $11 \%$, and growth retardation in $9 \%$.
Table 1. Baseline patients' characteristics

\begin{tabular}{lc}
\hline Characteristics & \\
\hline Gender (Feale/Male) & $59 / 77$ \\
Age at disease onset (years old) & $12.0(0.3-17.7)$ \\
Age at operation (years old) & $14.1(2.4-18.9)$ \\
Preoperative disease duration (days) & $679(16-3611)$ \\
& \\
Preoperative serum albumin (g/dL) & $3.2(1.4-4.8)$ \\
Preoperative serum hemoglobin (g/dL) & $10.6(4.9-17.5)$ \\
Preoperative serum CRP (mg/dL) & $0.33(0-16.11)$ \\
& \\
PUCAI & $45(0-85)$ \\
Japanese disease severity criteria & \\
$\quad$ Mild & $16(12 \%)$ \\
Moderate & $55(40 \%)$ \\
Severe & $61(45 \%)$ \\
unknown & $4(3 \%)$ \\
Montreal classification of UC & \\
E1 (ulcerative proctitis) & $0(0 \%)$ \\
E2 (left-sided UC) & $1(1 \%)$ \\
E3 (extensive UC) & $131(96 \%)$ \\
unknown & $4(3 \%)$ \\
\hline
\end{tabular}

$\overline{\mathrm{CRP} \text {, creactive protein; PUCAI, pediatric ulcerative colitis activity }}$ index; UC, ulcerative colitis. Data was shown by median (range).

Table 2. Operative indications

\begin{tabular}{lrr}
\hline Indication for operation & $n$ & $\%$ \\
\hline Perforation & 4 & 3 \\
Massive bleeding & 16 & 11 \\
Toxic Megacolon & 6 & 4 \\
Colon cancer & 0 & 0 \\
Fluminant disease & 4 & 3 \\
Growth Retardation & 13 & 9 \\
Intractable disease & 109 & 77 \\
Lower QOL & 33 & 23 \\
Drug side effect & 17 & 12 \\
Extra-intestinal complications & 2 & 1 \\
\hline
\end{tabular}

Table 3 shows the surgical procedures and relevant patient characteristics, including preoperative medications and laboratory data. Thirty-five patients had undergone one-stage surgery, 57 two-stage surgery, and 44 three-stage surgery. Patients who had undergone three-stage surgery had a significantly shorter duration of preoperative disease than those in the other groups ( $p$ $=0.0147)$; their more severe conditions being reflected by the necessity for more complex surgery $(p<0.0001)$, worse PUCAI scores at surgery $(p<0.0001)$, lower preoperative albumin $(p<0.0001)$ and hemoglobin concentrations ( $p=0.0059)$, and higher preoperative CRP concentrations $(p<0.0001)$. Preoperative medications varied by group and institution. In particular, few patients in this study received IFX.

SSI developed in 36/136 patients (26\%) (Table 4). I-SSI occurred in $28 / 136$ patients $(21 \%)$ and OS-SSI in $16 / 136$ patients $(12 \%)$. SSI occurred in $11 \%$ of patients who had undergone one-stage surgery, comprising I-SSI in $9 \%$ and OS-SSI in 9\%; in $19 \%$ patients who had undergone two-stage surgery, comprising I-SSI in $13 \%$ and OS-SSI in $12 \%$; and in $48 \%$ patients who had 
Table 3. Patients' characteristics among operative procedures

\begin{tabular}{|c|c|c|c|c|}
\hline Items & One & Two & Three & $p$ value \\
\hline girls : boys & $14: 21$ & $21: 35$ & $24: 20$ & 0.2055 \\
\hline Age at disease onset (years old) & $11.4 \pm 3.6$ & $11.4 \pm 3.1$ & $11.1 \pm 5.0$ & 0.7996 \\
\hline Severity at onset (mild/moderate/severe) & $3 / 21 / 8$ & $5 / 23 / 20$ & $4 / 16 / 20$ & \\
\hline PUCAI at disease onset & $52.5 \pm 25.4$ & $53.4 \pm 21.5$ & $62.2 \pm 21.2$ & 0.3496 \\
\hline Disease extent at onset (rectum/left side/total colitis) & $0 / 4 / 30$ & $3 / 5 / 41$ & $1 / 6 / 35$ & \\
\hline Age at operation (years old) & $14.0 \pm 2.7$ & $13.8 \pm 2.6$ & $13.1 \pm 3.6$ & 0.8278 \\
\hline Preoperative disease duration (days) & $951.6 \pm 782$ & $873.2 \pm 635.7$ & $657.1 \pm 834.1$ & 0.0147 \\
\hline Severity at operation (mild/moderate/severe) & $3 / 22 / 10$ & $11 / 26 / 16$ & $2 / 8 / 34$ & $<0.0001$ \\
\hline PUCAI at operation & $38.9 \pm 20.5$ & $39.5 \pm 25.8$ & $69.0 \pm 21.6$ & $<0.0001$ \\
\hline Disease extent at operation (E1/E2/E3) & $0 / 1 / 33$ & $0 / 1 / 54$ & $0 / 1 / 43$ & 0.5627 \\
\hline Preoperative serum albumin (g/dL) & $3.7 \pm 0.8$ & $3.7 \pm 0.6$ & $2.7 \pm 0.8$ & $<0.0001$ \\
\hline Preoperative serum hemoglobin (g/dL) & $11.5 \pm 2.3$ & $11.2 \pm 2.3$ & $9.7 \pm 2.2$ & 0.0059 \\
\hline Preoperative serum CRP $(\mathrm{mg} / \mathrm{dL})$ & $1.9 \pm 3.7$ & $0.8 \pm 2.2$ & $4.0 \pm 4.5$ & $<0.0001$ \\
\hline Preoperative 90 5ASA (y/n) & $31 / 2$ & $53 / 4$ & $28 / 11$ & 0.0064 \\
\hline Preoperative 90 PDN (y/n) & $32 / 2$ & $42 / 15$ & $32 / 7$ & 0.0344 \\
\hline Preoperative 90 APT (y/n) & $13 / 19$ & $15 / 42$ & $8 / 31$ & 0.1665 \\
\hline Preoperative 90 AZA (y/n) & $11 / 21$ & $30 / 27$ & $8 / 31$ & 0.0047 \\
\hline Preoperative 90 TAC $(\mathrm{y} / \mathrm{n})$ & $3 / 29$ & $17 / 40$ & $5 / 34$ & 0.0277 \\
\hline Preoperative 90 CYA (y/n) & $3 / 29$ & $13 / 44$ & $10 / 29$ & 0.1589 \\
\hline Preoperative $90 \operatorname{IFX}(\mathrm{y} / \mathrm{n})$ & $1 / 31$ & $9 / 48$ & $1 / 38$ & 0.0288 \\
\hline Preoperative 30 5ASA (y/n) & $30 / 3$ & $49 / 8$ & $26 / 16$ & 0.003 \\
\hline Preoperative $30 \mathrm{PDN}(\mathrm{y} / \mathrm{n})$ & $31 / 3$ & $39 / 18$ & $38 / 6$ & 0.0131 \\
\hline Preoperative 30 APT (y/n) & $9 / 23$ & $8 / 49$ & $13 / 29$ & 0.0935 \\
\hline Preoperative 30 AZA (y/n) & $12 / 20$ & $24 / 33$ & $7 / 35$ & 0.0179 \\
\hline Preoperative 30 TAC $(y / n)$ & $4 / 28$ & $13 / 44$ & $6 / 36$ & 0.3762 \\
\hline Preoperative 30 CYA (y/n) & $3 / 29$ & $12 / 45$ & $9 / 33$ & 0.2805 \\
\hline Preoperative $30 \operatorname{IFX}(\mathrm{y} / \mathrm{n})$ & $1 / 31$ & $1 / 56$ & $1 / 41$ & 0.9182 \\
\hline $\operatorname{EsPDN}(g)$ & $633.5 \pm 751.0$ & $663.7 \pm 1004.7$ & $838.2 \pm 839.4$ & 0.87 \\
\hline $\operatorname{EsPDN}(\mathrm{g}) / \mathrm{BW}(\mathrm{kg})$ & $14.7 \pm 15.9$ & $21.8 \pm 34.9$ & $22.0 \pm 22.5$ & 0.1586 \\
\hline
\end{tabular}

CRP; creactive protein, PUCAI; pediatric ulcerative colitis activity index, 5ASA; 5-aminosalicylates, PDN; prednisolone, APT; apheresis therapy, AZA; azathioprine, TAC; tacrolimus, CYA; cyclosporine A, IFX; infliximab, y; yes, n; no, EsPDN; estimated PDN administered dose during 1 month before operation, BW; body weight. Data was shown by mean \pm standard deviation.

Table 4. SSI occurrence among operative procedures

\begin{tabular}{lllcr}
\hline Items & Total (136) & One (35) & Two (57) & Three (44) \\
\hline SSI & $36(26 \%)$ & $4(11 \%)$ & $11(19 \%)$ & $21(48 \%)$ \\
I-SSI & $28(21 \%)$ & $3(9 \%)$ & $8(13 \%)$ & $17(35 \%)$ \\
OS-SSI & $16(12 \%)$ & $3(9 \%)$ & $7(12 \%)$ & $6(14 \%)$ \\
\hline
\end{tabular}

SSI; surgical site infection, I-SSI; incisional wound infection, OSSSI; organ/space infection.

undergone three-stage surgery, comprising I-SSI in 35\% and OS-SSI in $14 \%$.

Table 5 shows the relationships between development of SSI after first stage operations and selected factors. SSI occurred significantly more frequently in girls than boys $(p=0.012)$. Neither disease severity nor preoperative medications were significantly associated with development of SSI. There was a statistically significant relationship between staged surgery and development of SSI ( $p=0.0004)$.

Table 6 shows the results of multiple logistic regression analysis. Staged surgery (three/one, OR: 6.7, 95\% CI: 2.1-25.5, $p=0.0007$; three/two, OR: $3.4,95 \%$ CI: $1.4-8.6, p=0.0069$ ) and female sex (OR: 2.3, 95\% CI: $1.0-5.4, p=0.0434)$ were statistically significant predictors of development of SSI in pediatric patients with UC. Staged surgery was the sole statistically significant risk factor for I-SSI, (three/one, OR: 6.5, 95\% CI: $1.9-30.2, p=0.0021$; three/two, OR: $3.6,95 \% \mathrm{CI}$ : $1.4-9.9, p=0.0091$ ); however, no predictors of OS-SSI, such as anastomotic leakage or intraperitoneal abscess, were identified in this study.

\section{Discussion}

The major findings of this study were that female sex and three-stage surgery are independent predictors of development of SSI after surgery for UC in pediatric patients. Three-stage surgery was an independent predictor of development of I-SSI occurrence; female sex was not. No predictors of OS-SSI were identified. Preoperative medications, including 5-ASA, AZA, TAC, and CYA, did not significantly affect the incidence of SSI.

SSI is the most frequent complication of colorectal surgery. In adult colorectal surgery, dirty or contaminated surgery, open ileostomy or colostomy placement, emergency surgery, and multiple procedures are reportedly associated with increased risk of SSI $(17,18)$. Segal et al. (19) reported that risk factors for I-SSI and OS-SSI differ in patients who undergo colon resection.

The surgical management for UC is challenging because the procedure of IPAA is difficult; additionally, 
Table 5. The relationship between SSI occurrence and several factors in patients who received first stage operation

\begin{tabular}{lccc}
\hline Items & SSI (+) & SSI (-) & $p$ value \\
\hline girls : boys & $22: 14$ & $37: 63$ & 0.0125 \\
Age at disease onset (years old) & $11.4 \pm 4.3$ & $11.3 \pm 3.7$ & 0.6570 \\
Severity at onset (mild/moderate/severe) & $2 / 15 / 16$ & $6 / 49 / 32$ & 0.5065 \\
PUCAI at disease onset & $58.3 \pm 26.2$ & $54.9 \pm 20.3$ & 0.5956 \\
Disease extent at onset (rectum/left side/total colitis) & 36674 & $4 / 10 / 78$ & 0.2459 \\
Age at operation (years old) & $13.9 \pm 2.2$ & $13.5 \pm 3.2$ & 0.7962 \\
Preoperative disease duration (days) & $864.9 \pm 980.6$ & $811.0 \pm 642.9$ & 0.4544 \\
Severity at operation (mild/moderate/severe) & $5 / 14 / 16$ & 0.9492 \\
PUCAI at operation & $53.7 \pm 28.6$ & $12 / 41 / 44$ & 0.1828 \\
Disease extent at operation (E1/E2/E3) & $0 / 0 / 35$ & $45.0 \pm 26.1$ & 0.3952 \\
Preoperative serum albumin (g/dL) & $3.2 \pm 0.8$ & $1 / 2 / 95$ & 0.1087 \\
Preoperative serum hemoglobin (g/dL) & $10.4 \pm 2.7$ & $3.4 \pm 0.9$ & 0.1998 \\
Preoperative serum CRP (mg/dL) & $2.4 \pm 4.0$ & $11.0 \pm 2.2$ & 0.0927 \\
Preoperative 90 5ASA (y/n) & $27 / 6$ & $1.9 \pm 3.5$ & 0.4716 \\
Preoperative 90 PDN (y/n) & $26 / 7$ & $85 / 12$ & 0.6412 \\
Preoperative 90 APT (y/n) & $9 / 24$ & $80 / 17$ & 0.8992 \\
Preoperative 90 AZA (y/n) & $12 / 21$ & $27 / 68$ & 0.7921 \\
Preoperative 90 TAC (y/n) & $6 / 27$ & $37 / 58$ & 0.8194 \\
Preoperative 90 CYA (y/n) & $6 / 27$ & $19 / 76$ & 0.7216 \\
Preoperative 90 IFX (y/n) & $1 / 32$ & $20 / 75$ & 0.1453 \\
Preoperative 30 5ASA (y/n) & $25 / 9$ & $10 / 85$ & 0.3226 \\
Preoperative 30 PDN (y/n) & $29 / 7$ & $80 / 18$ & 0.9223 \\
Preoperative 30 APT (y/n) & $8 / 26$ & $79 / 20$ & 0.8211 \\
Preoperative 30 AZA (y/n) & $9 / 25$ & $21 / 76$ & 0.4761 \\
Preoperative 30 TAC (y/n) & $6 / 28$ & $32 / 65$ & 0.9872 \\
Preoperative 30 CYA (y/n) & $5 / 29$ & $17 / 80$ & 0.3706 \\
Preoperative 30 IFX (y/n) & $0 / 34$ & $21 / 76$ & 0.1764 \\
EsPDN(g) & $843.5 \pm 869.9$ & $3 / 94$ & 0.0917 \\
EsPDN(g) / BW (kg) & $24.8 \pm 29.4$ & 0.1800 \\
Stage operation (one/two/three) & $4 / 11 / 21$ & 0.0004 \\
\hline & & $652.0 \pm 915.9$ & \\
SSI sich & & $31 / 46 / 23$ & \\
\end{tabular}

SSI; surgical site infection, CRP; creactive protein, PUCAI; pediatric ulcerative colitis activity index, 5ASA; 5-aminosalicylates, PDN; prednisolone, APT; apheresis therapy, AZA; azathioprine, TAC; tacrolimus, CYA; cyclosporine A, IFX; infliximab, y; yes, n; no, EsPDN; estimated PDN administered dose during 1month before operation, BW; body weight. Data was shown by mean \pm standard deviation.

Table 6. Multiple logistic regression analysis

\begin{tabular}{|c|c|c|c|c|c|}
\hline \multirow{2}{*}{ Items } & \multirow{2}{*}{$\begin{array}{c}\text { Chi square } \\
p \text { value }\end{array}$} & & \multicolumn{3}{|c|}{ Multiple logistic regression analysis } \\
\hline & & & OR & $95 \% \mathrm{CI}$ & $p$ value \\
\hline \multicolumn{6}{|l|}{ SSI } \\
\hline female & 0.0141 & two/one & 2.3 & $1.0-5.4$ & 0.0434 \\
\hline \multirow[t]{3}{*}{ staged operation } & 0.0004 & three/one & 2 & $0.6-7.7$ & 0.272 \\
\hline & & three/two & 6.7 & $2.1-25.5$ & 0.0007 \\
\hline & & & 3.4 & $1.4-8.6$ & 0.0069 \\
\hline \multicolumn{6}{|l|}{ I-SSI } \\
\hline female & 0.0460 & & & & 0.132 \\
\hline \multirow[t]{3}{*}{ staged operation } & 0.0012 & two/one & 1.8 & $0.5-8.9$ & 0.3888 \\
\hline & & three/one & 6.5 & $1.9-30.2$ & 0.0021 \\
\hline & & three/two & 3.6 & $1.4-9.9$ & 0.0091 \\
\hline $\begin{array}{r}\text { OS-SSI } \\
\text { none }\end{array}$ & & & & & \\
\hline
\end{tabular}

SSI; surgical site infection, I-SSI; incisional wound infection, OS-SSI; organ/space infection.

patients with UC tend to be in poorer condition than patients with other colorectal diseases requiring surgery because of infection or contamination, proinflammatory factors characteristic of $\mathrm{UC}$, anemia and malnutrition, and immunosuppression, including that induced by immunosuppressive medication. Patients with UC undergoing elective colectomy reportedly have significantly higher rates of SSI, especially OS-SSI $(5,6)$. Araki et al. (20) have reported that poor general physical status is a significant independent risk factor for OS-SSI in adult patients with UC. In a comparison of colorectal surgery for colorectal cancer versus IBD in patients with clean-contaminated wounds, IBD was shown to be an independent risk factor for I-SSI ( 6 ). 
In this study, surgery for UC was performed in one stage in 35 cases, two stages in 57 cases, and three stages in 44 cases. We found three-stage surgery to be a statistically significant independent predictor of SSI, likely because of risk factors such as dirty or contaminated surgery, creation of ileostomies, and the characteristically poor condition of patients requiring three-stage surgery compared with that of those requiring only one- or two- stage surgery.

We found that female sex is a predictor of SSI, as shown by two other studies $(17,21)$ of colorectal surgery for adult patients. In contrast, Morikane et al. (18) found an association between male sex and increased risk of SSI in adult patients undergoing colorectal surgery. The reasons for SSI being more common in female than male patients in three of these four studies is unclear; however, Pedroso-Fernandez et al. (17) proposed in a personal communication that the procedure for extracting the resected tissue and size of incision may differ in female patients for cosmetic reasons.

Until ten years ago, high total PDN doses were administered before surgery and this was strongly associated with incidence of SSI in patients with UC, especially in Japanese institutes (22-25). Use of nonsteroid drugs, including immunomodulators and antitumor necrosis factor, has recently been increasing in pediatric patients with a hopeful future, though corticosteroids remain the main drug for inducing remission of UC. If the medications used instead of PDN for treating UC have immunosuppressive effects that may lead to infectious complications, such drugs could increase the incidence of surgical complications. Thus far, several studies have concluded that there is no relationship between preoperatively administered medications and postoperative SSI in pediatric patients with UC (25-29). Regarding use of immunomodulators, preoperative exposure to thioprines or calcineurin inhibitors is reportedly not associated with increased postoperative complications in pediatric patients with UC who undergo colectomy $(27,29)$. Patton et al. (28) found that preoperative IFX was not associated with postoperative complications in pediatric patients with UC. However, Kennedy et al. (26) reported that postoperative small bowel obstruction occurred more frequently in children who had been treated with IFX prior to proctocolectomy than in those who had not; however, this relationship is unclear. Because too few patients in our study had received IFX, our results did not allow us to draw a conclusion about any relationship between preoperative IFX administration and SSI occurrence.

This study has some limitations. Three-stage surgery was selected for pediatric patients with perforation, toxic megacolon, and IBD-unclassified whereas selection of one- or two-stage surgery was dependent on the policy and judgement within each surgical institute and the obtaining of informed consent for proposed procedures.
Additionally, precautions taken to prevent SSI, surgical indications and procedures, and length and positions of incisions differed between the surgical institutes. Concerning the relationship between preoperatively administered medications and SSI, we did not monitor 6-thioguanine nucleotide concentrations in patients receiving AZA (30) or trough serum con/ss in those receiving TAC (31), CYA (32), or IFX (33) in this study; we therefore could not investigate the relationship between serum concentrations of drugs and postoperative infectious complications.

In conclusion, three-stage surgery is an independent predictor of development of SSI after surgery in pediatric patients with UC. In this study, preoperative medication, including 5-ASA, AZA, TAC, and CYA, did not affect the overall incidence of SSI, or that of I-SSI or OS-SSI. An immediate correct diagnosis, appropriate medical therapy without using high total doses of PDN, optimal timing of surgery, and avoidance of three-stage surgery may result in better surgical outcomes and quality of life.

\section{Acknowledgements}

This study was supported by a grant-in-aid for Intractable Disease from the Ministry of Health, Labour, and Welfare of Japan.

The authors thank those who participated in the Ministry of Health and Welfare of Japan's Inflammatory Bowel Diseases Study Group, especially the Department of Gastroenterology and Hepatology, Tokyo Medical and Dental University (Mamoru Watanabe), Department of Internal Medicine, Sakura Medical Center, Toho University (Yasuo Suzuki), Department of Inflammatory Bowel Disease Surgery, Hyogo College of Medicine (Hiroki Ikeuchi and Motoi Uchino), Department of Surgery, Yokohama Municipal Hospital (Akira Sugita and Kazutaka Koganei), Inflammatory Bowel Disease Center, Yokohama City University Medical Center (Reiko Kunisaki), and Center for Advanced IBD Research and Treatment, Kitasato Institute Hospital, Kitasato University (Toshifumi Hibi) for their contributions to this work.

\section{References}

1. Ishige $\mathrm{T}$, Tomomasa $\mathrm{T}$, Takebayashi $\mathrm{T}$, Asakura $\mathrm{K}$, Watanabe M, Suzuki T, Miyazawa R, Arakawa H. Inflammatory bowel disease in children: Epidemiological analysis of the nationwide IBD registry in Japan. J Gastroenterol. 2010; 45:911-917.

2. Soon IS, deBruyn JC, Hubbard J, Wrobel I, Sauve R, Sigalet DL, Kaplan GG. Rising post-colectomy complications in children with ulcerative colitis despite stable colectomy rates in United States. J Crohns Colitis. 2014; 8:1417-1426.

3. Soon IS, Wrobel I, deBruyn JC, Sauve R, Sigalet DL, Kaplan BS, Proulx MC, Kaplan GG. Postoperative complications following colectomy for ulcerative colitis in 
children. J Pediatr Gastroenterol Nutr. 2012; 54:763-768.

4. Knod JL, Holder M, Cortez AR, Martinez-Leo B, Kern P, Saeed S, Warner B, Dickie B, Falcone RA, von Allmen D, Frischer JS. Surgical outcomes, bowel habits and quality of life in young patients after ileoanal anastomosis for ulcerative colitis. J Pediatr Surg. 2016; 51:1246-1250.

5. Bhakta A, Tafen M, Glotzer O, Ata A, Chismark AD, Valerian BT, Stain SC, Lee EC. Increased Incidence of Surgical Site Infection in IBD Patients. Dis Colon Rectum. 2016; 59:316-322.

6. Uchino M, Ikeuchi H, Tsuchida T, Nakajima K, Tomita N, Takesue Y. Surgical site infection following surgery for inflammatory bowel disease in patients with cleancontaminated wounds. World J Surg. 2009; 33:1042-1048.

7. Øresland T, Bemelman WA, Sampietro GM, et al. European Crohn's and Colitis Organisation (ECCO). European evidence based consensus on surgery for ulcerative colitis. J Crohns Colitis. 2015; 9:4-25.

8. Mahadevan U, Loftus EV Jr, Tremaine WJ, Pemberton JH, Harmsen WS, Schleck CD, Zinsmeister AR, Sandborn WJ. Azathioprine or 6-mercaptopurine before colectomy for ulcerative colitis is not associated with increased postoperative complications. Inflamm Bowel Dis. 2002; 8:311-316.

9. Aberra FN, Lewis JD, Hass D, Rombeau JL, Osborne B, Lichtenstein GR. Corticosteroids and immunomodulators: Postoperative infectious complication risk in inflammatory bowel disease patients. Gastroenterology. 2003; 125:320327.

10. Hosoi K, Ohtsuka Y, Fujii T, et al. The treatment with infliximab for pediatric Crohn's disease: Nationwide survey of Japan. J Gastroenterol Hepatol. 2017; 32:114119.

11. IBD Working Group of the European Society for Paediatric Gastroenterology, Hepatology and Nutrition. Inflammatory bowel disease in children and adolescents: Recommendations for diagnosis - the Porto criteria. J Pediatr Gastroenterol Nutr. 2005; 41:1-7.

12. Turner D, Otley AR, Mack D, Hyams J, de Bruijne J, Uusoue K, Walters TD, Zachos M, Mamula P, Beaton DE, Steinhart AH, Griffiths AM. Development, validation, and evaluation of a Pediatric Ulcerative Colitis Activity Index: A prospective multicenter study. Gastroenterology. 2007; 133:423-432.

13. Hiwatashi $\mathrm{N}$. The diagnostic criteria for determination of ulcerative colitis severity. In: The 1993 Annual Report of the Research Committee of Inflammatory Bowel Disease. Ministry of Health and Welfare of Japan, Tokyo, Japan, 1994; pp. 90-92.

14. Satsangi J, Silverberg MS, Vermeire S, Colombel JF. The Montreal classification of inflammatory bowel disease: Controversies, consensus, and implications. Gut. 2006; 55:749-53.

15. Mangram AJ, Horan TC, Pearson ML, Silver LC, Jarvis WR. Guideline for prevention of surgical site infection, 1999 Centers for Disease Control and Prevention (CDC) Hospital Infection Control Practices Advisory Committee. Am J Infect Control. 1999; 27:97-132.

16. Pendlimari R, Cima RR, Wolff BG, Pemberton JH, Huebner M. Diagnoses influence surgical site infections (SSI) in colorectal surgery: A must consideration for SSI reporting programs? J Am Coll Surg. 2012; 214:574-580.

17. Pedroso-Fernandez Y, Aguirre-Jaime A, Ramos MJ, Hernández M, Cuervo M, Bravo A, Carrillo A. Prediction of surgical site infection after colorectal surgery. Am J
Infect Control. 2016; 1:450-454

18. Morikane K, Honda H, Yamagishi T, Suzuki S, Aminaka M. Factors associated with surgical site infection in colorectal surgery: The Japan nosocomial infections surveillance. Infect Control Hosp Epidemiol. 2014; 35:660-666.

19. Segal CG, Waller DK, Tilley B, Piller L, Bilimoria K. An evaluation of differences in risk factors for individual types of surgical site infections after colon surgery. Surgery. 2014; 156:1253-1260.

20. Araki T, Okita Y, Uchino M, et al. Risk factors for surgical site infection in Japanese patients with ulcerative colitis: A multicenter prospective study. Surg Today. 2014; 44:10721078.

21. Blitzer DN, Davis JM, Ahmed N, Kuo YH, Kuo YL. Impact of procedure on the post-operative infection risk of patients after elective colon surgery. Surg Infect (Larchmt). 2014; 15:721-725.

22. Kusunoki M, Möeslein G, Shoji Y, Fujita S, Yanagi H, Sakanoue Y, Saito N, Utsunomiya J. Steroid complications in patients with ulcerative colitis. Dis Colon Rectum. 1992; 35:1003-1009.

23. Shinozaki M, Suzuki K, Sawada T, Tsuno N, Higuchi Y, Muto T. Steroid complications and surgery in intractable ulcerative colitis. J Gastroenterol. 1998; 33:196-200.

24. Uchida K, Araki T, Toiyama Y, Yoshiyama S, Inoue M, Ikeuchi H, Yanagi H, Miki C, Yamamura T, Kusunoki M. Preoperative steroid-related complications in Japanese pediatric patients with ulcerative colitis. Dis Colon Rectum. 2006; 49:74-79.

25. Markel TA, Lou DC, Pfefferkorn M, Scherer LR 3rd, West K, Rouse T, Engum S, Ladd A, Rescorla FJ, Billmire DF. Steroids and poor nutrition are associated with infectious wound complications in children undergoing first stage procedures for ulcerative colitis. Surgery. 2008; 144:540545.

26. Kennedy R, Potter DD, Moir C, Zarroug AE, Faubion W, Tung J. Pediatric chronic ulcerative colitis: Does infliximab increase post-ileal pouch anal anastomosis complications? J Pediatr Surg. 2012; 47:199-203.

27. Schaufler C, Lerer T, Campbell B, Weiss R, Cohen J, Sayej W, Hyams J. Preoperative immunosuppression is not associated with increased postoperative complications following colectomy in children with colitis. J Pediatr Gastroenterol Nutr. 2012; 55:421-424.

28. Patton D, Gupta N, Wojcicki JM, Garnett EA, Nobuhara K, Heyman MB. Postoperative outcome of colectomy for pediatric patients with ulcerative colitis. J Pediatr Gastroenterol Nutr. 2010; 51:151-154.

29. Hait EJ, Bousvaros A, Schuman M, Shamberger RC, Lillehei CW. Pouch outcomes among children with ulcerative colitis treated with calcineurin inhibitors before ileal pouch anal anastomosis surgery. J Pediatr Surg. 2007; 42:31-34.

30. Ohtsuka Y, Arai K, Aoyagi Y, Fujii T, Yamakawa Y, Ohtani K, Ikuse T, Baba Y, Inage E, Kudo T, Suzuki R, Nagata S, Shimizu T. Monitoring 6-thioguanine nucleotide concentrations in Japanese children and adolescents with inflammatory bowel disease. J Gastroenterol Hepatol. 2010; 25:1626-1630.

31. Navas-López VM, Blasco Alonso J, Serrano Nieto MJ, Girón Fernández-Crehuet F, Argos Rodriguez MD, Sierra Salinas C. Oral tacrolimus for pediatric steroid-resistant ulcerative colitis. J Crohns Colitis. 2014; 8:64-69.

32. Benkov KJ, Rosh JR, Schwersenz AH, Janowitz HD, 
LeLeiko NS. Cyclosporine as an alternative to surgery in children with inflammatory bowel disease. J Pediatr Gastroenterol Nutr. 1994; 19:290-294.

33. Singh N, Rosenthal CJ, Melmed GY, Mirocha J, Farrior S, Callejas S, Tripuraneni B, Rabizadeh S, Dubinsky MC. Early infliximab trough levels are associated with persistent remission in pediatric patients with inflammatory bowel disease. Inflamm Bowel Dis. 2014; 20:1708-1713.

(Received March 1, 2017; Revised March 26, 2017; Accepted April 2, 2017) 